\title{
Emissão por folhas de Ficus benjamina L. (Moraceae) de compostos orgânicos voláteis oxigenados
}

\author{
SILVIA R. SOUZA ${ }^{1}$, PÉROLA C. VASCONCELLOS ${ }^{2}$, \\ WALDIR MANTOVANI $^{3}$ e LILIAN R.F. CARVALHO ${ }^{1,4}$
}

(recebido: 20 de setembro de 2001; aceito: 19 de junho de 2002)

\begin{abstract}
Oxygenated volatile organic compounds emitted from leaves of Ficus benjamina L. (Moraceae)). Vegetation is the source of over $90 \%$ of all volatile organic compounds in the global atmosphere. Some oxygenated hydrocarbons emitted by plants react with radicals, such as nitrate and hydroxyl, and ozone at comparable rates to those of the most reactive anthropogenic compounds and they can contribute to ozone formation in urban areas. Although the natural hydrocarbons have an important role in the formation of photochemical oxidant, chemical speciation of the oxygenated volatile organic compounds is still very limited. In this paper, enclosured gaseous samples were collected from Ficus benjamina, a common plant species in the region of the São Paulo City, and various oxygenated volatile organic compounds, such as organic acids (formic and acetic acids), aldehydes (formaldehyde, acetaldehyde and hexanal) and alcohols (menthol, 1- butanol, 1-pentanol, 2-penten-1-ol, 4-penten-2-ol e linalool), were identified by using chromatographic techniques.

RESUMO - (Compostos orgânicos voláteis oxigenados emitidos pelas folhas de Ficus benjamina L. (Moraceae)). A vegetação é a fonte de aproximadamente $90 \%$ de todos os compostos orgânicos voláteis na atmosfera global. Alguns hidrocarbonetos oxigenados emitidos por plantas reagem com radicais livres, tais como nitrato e hidroxila, e ozônio em taxas comparáveis com aquelas dos compostos antropogênicos mais reativos e podem contribuir para a formação de ozônio em áreas urbanas. Apesar do papel importante dos hidrocarbonetos naturais na formação fotoquímica dos oxidantes, pouco se sabe sobre as espécies químicas dos compostos orgânicos voláteis emitidos por plantas. Nesse trabalho, foi estudada a emissão de compostos orgânicos voláteis por Ficus benjamina, espécie comumente encontrada na região da cidade de São Paulo. Os gases emitidos pelas folhas da $F$. benjamina foram coletados em sistemas fechados e vários compostos orgânicos voláteis oxigenados, tais como ácidos orgânicos (ácido fórmico e ácido acético), aldeídos (formaldeído, acetaldeído e hexanal) e álcoois (mentol, 1- butanol, 1-pentanol, 2-penten-1-ol, 4-penten-2-ol e linalool), foram identificados através de técnicas cromatográficas.
\end{abstract}

Key words - Biogenic atmospheric emissions, Ficus benjamina, oxygenated hydrocarbons, volatile organic compounds

\section{Introdução}

Estima-se que as emissões de compostos orgânicos voláteis (COVs) pela vegetação são comparáveis, ou excedem, as emissões de COVs de fontes antropogênicas, em escala regional e global (Guenther 1999). Os compostos orgânicos voláteis incluem os hidrocarbonetos não-metânicos saturados e insaturados e os hidrocarbonetos oxigenados, tais como ácidos carboxílicos, aldeídos, cetonas, éteres, ésteres e álcoois (Seinfeld \& Pandis 1998).

Um grande número de compostos oxigenados tem sido encontrado nas emissões de plantas. Os inventários de emissão de plantas mostram que isopreno e

\footnotetext{
1. Universidade de São Paulo, Instituto de Química, Av. Prof. Lineu Prestes, 748, 05508-900 São Paulo, SP, Brasil.

2. Instituto de Pesquisas Energéticas e Nucleares, Travessa "R", 400, 05508-900 São Paulo, SP, Brasil.

3. Universidade de São Paulo, Instituto de Biociências, Caixa Postal 11461, 05422-970 São Paulo, SP, Brasil.

4. Autor para correspondência: Irfdcarv@iq.usp.br
}

monoterpenos, classificados como isoprenóides, são os compostos mais abundantes, seguidos de álcoois e carbonilas (Tingey et al. 1991). Em geral, a maioria dos COVs pode sofrer fotólise quando irradiados pela luz solar ou reagir com ozônio e radicais $\mathrm{OH}$ e $\mathrm{NO}_{3}$ (Seinfeld \& Pandis 1998).

Concentrações atmosféricas de ozônio e outros poluentes do ar, em algumas regiões, são sensíveis a fluxos superficiais de COVs. Os COVs reagem com o ozônio mas também geram ozônio na presença de $\mathrm{NO}_{\mathrm{x}}$ e o balanço global de ozônio depende principalmente da quantidade de $\mathrm{NO}_{\mathrm{x}}$ presente na atmosfera (Ciccioli 1993). Como os COVs biogênicos reagem com as espécies reativas radicalares e o ozônio na mesma taxa que a dos compostos antropogênicos mais reativos, os COVs biogênicos contribuem para a formação de ozônio em áreas onde estão presentes quantidades suficientes de $\mathrm{NO}_{\mathrm{x}}$ (Guenther et al. 1995, Grosjean 1995). Dados obtidos por modelos matemáticos evidenciaram que as emissões pela vegetação têm um papel importante na química da troposfera, podendo 
contribuir na produção de ozônio em áreas urbanas e rurais (Guenther et al. 1995).

Muitos dos COVs são altamente reativos e estão envolvidos na química da troposfera, afetando o balanço regional e global de oxidantes e contribuindo para a produção e deposição úmida de ácidos orgânicos (Fehsenfeld et al. 1992). Apesar de as emissões antropogênicas serem dominantes em áreas urbanas e industriais, nos Estados Unidos, cerca da metade dos COVs emitidos origina-se de fontes naturais (Guenther et al. 1995). As fontes desses compostos são variáveis em tempo e espaço e as estimativas das emissões permanecem incertas.

Estudos para avaliar a liberação de COVs por plantas são ainda insuficientes frente ao elevado número de espécies de plantas e à grande diversidade das espécies químicas emitidas para a atmosfera. A estimativa do fluxo de vários compostos de uma dada região requer um conhecimento detalhado da composição química dos gases emitidos pela vegetação que contém. Apesar de pouco conhecida, as emissões pela vegetação nas regiões tropicais devem ter um papel importante no comportamento atmosférico, uma vez que nessas regiões existe uma ampla diversidade de espécies de plantas e uma grande complexidade da vegetação. Recentemente, estudos têm sido direcionados para a identificação dos compostos orgânicos voláteis oxigenados, incluindo metanol (McDonald \& Fall 1993a, Nemecek-Marshall et al. 1995), derivados de hexanal (Arey et al. 1991), acetona (McDonald \& Fall 1993b), alguns ácidos orgânicos (Talbot et al. 1990) e aldeídos (Kesselmeier \& Staudt 1999). Câmaras fechadas têm sido freqüentemente empregadas para coletar os gases emitidos pelas plantas (Harley et al. 1998, Kesselmeier \& Staudt 1999). Uma câmara fechada acoplada a um coletor de gases permite amostrar e pré-concentrar COVs emitidos diretamente pela planta.

COVs, tais como monoterpenos, sesquiterpenos, álcoois, ácidos, aldeídos, cetonas e ésteres estão armazenados nas plantas em diferentes órgãos. As plantas, que durante a fotossíntese fixam de 0,5 a $2 \%$ do carbono, emitem taxas elevadas de COVs, provocando a perda de até $20 \%$ do carbono fixado (Harley et al. 1998). Acredita-se que tais compostos são emitidos para a atmosfera como mecanismo de defesa contra o ataque de herbívoros e contra a invasão de outras espécies de plantas (Tingey et al. 1991).

Várias espécies de plantas que emitem uma quantidade significativa de isopreno e monoterpenos foram estudadas em alguns países do mundo (Winer et al. 1992, Klinger et al. 1998, Kesselmeier \& Staudt 1999) e alguns gêneros dessas plantas existem na Floresta Ombrófila Densa em São Paulo, Brasil (Aragaki \& Mantovani 1998). Por outro lado, existem espécies abundantes no Planalto Atlântico, recentemente estudadas na Amazônia, que emitem quantidades significativas de COVs (P. C. Vasconcellos, dados não publicados). No presente trabalho, os gases emitidos por Ficus benjamina L., uma espécie abundante na região da cidade de São Paulo (Rossi 1994, Aragaki \& Mantovani 1998), foram analisados quimicamente para caracterizar a emissão dos COVs oxigenados. Esse estudo faz parte de um projeto de pesquisa amplo envolvendo emissões de COVs provenientes da vegetação em São Paulo, os quais podem afetar a formação de oxidantes fotoquímicos na Região Metropolitana de São Paulo.

\section{Material e métodos}

No presente trabalho, a planta estudada foi a Ficus benjamina L. (Moraceae), uma espécie originária da Malásia e Índia. Os exemplares estudados foram adquiridos de um produtor comercial. Os gases emitidos pelas folhas da $F$. benjamina foram coletados em sistemas fechados e a especiação química dos COVs oxigenados foi feita empregando métodos cromatográficos.

Coleta dos gases - Para os seis experimentos realizados em laboratório, foi escolhido um exemplar jovem de $F$. benjamina cultivado em vaso, sendo que ramos distintos da planta foram usados para o confinamento das folhas. Para avaliar as emissões de COVs da planta em estudo foram empregados sistemas fechados estático e dinâmico (Tingey et al. 1991). O sistema estático consistiu em introduzir as folhas vivas da planta em um saco de polietileno (figura 1), previamente tratado com metanol para eliminação de possíveis impurezas e preenchido com ar isento de COVs. Após 30, 60 e 90 min de permanência das folhas no saco fechado hermeticamente, os gases emitidos foram coletados para análise. Por três vezes, transferiu-se, com auxílio de uma seringa, 1,5 L do ar contido no saco para recipientes de vidro, "impingers", contendo separadamente, $5,0 \mathrm{~mL}$ de metanol, solução de dinitrofenil-hidrazina em acetonitrila, 1,0 g.L. $\mathrm{L}^{-1}$, e solução aquosa $5 \%$ de carbonato de sódio para reter respectivamente álcoois, carbonílicos e ácidos carboxílicos. Após amostragem do ar e retenção dos compostos nas soluções, as mesmas foram submetidas às análises químicas empregando técnicas cromatográficas. Ácidos carboxílicos, carbonílicos e álcoois foram analisados por cromatografia de íons, cromatografia a líquido e cromatografia a gás, respectivamente.

Paralelamente aos testes feitos em sistema estático, uma câmara fechada ("cuvette") foi construída para coletar os 


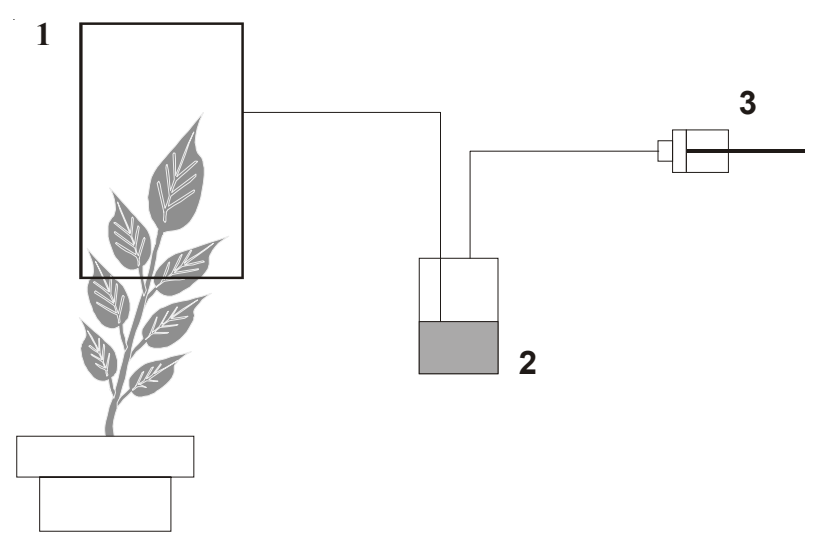

Figura 1. Sistema estático para coleta de gases provenientes das emissões de plantas. ( $1=$ saco de polietileno preenchido com ar; 2 = recipiente contendo solução pré-concentradora; $3=$ seringa para sucção do ar).

gases em sistema dinâmico. A aparelhagem usada para o confinamento de folhas da planta viva foi constituída de uma câmara cilíndrica construída em Teflon ${ }^{\circledR}$ e com volume interno igual a $720 \mathrm{~cm}^{3}$, contendo dois orifícios laterais para a entrada e saída do ar e uma tampa com visor em vidro para incidência de luz sobre as folhas de Ficus benjamina. Uma bomba de sucção foi empregada para fazer o ar atravessar a câmara fechada, em seguida, um coletor para reter os compostos de interesse (figura 2). Um fluxo contínuo de ar isento de COVs foi injetado na câmara durante o período da coleta. Os compostos voláteis emitidos pela planta foram retidos na saída da câmara em um tubo de vidro contendo um adsorvente sólido (Porapack). Utilizou-se uma vazão de $200 \mathrm{~mL} / \mathrm{min}$ e o tempo de amostragem foi de uma hora. Após a coleta, o cartucho contendo o adsorvente sólido foi submetido à extração com 5,0 mL de diclorometano. $\mathrm{O}$ extrato orgânico foi analisado por cromatografia a gás.

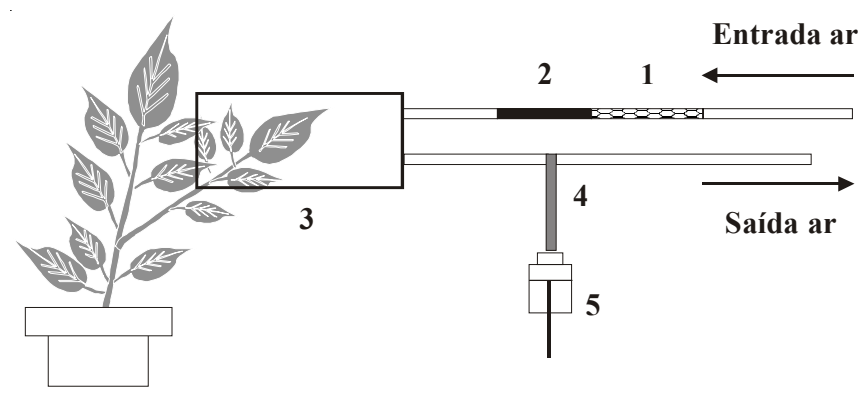

Figura 2. Sistema dinâmico para coleta de gases provenientes das emissões de plantas. ( 1 = cartucho contendo adsorvente sólido (Porapack) para eliminar COVs do ar; 2 = cartucho contendo sílica para eliminar a umidade; 3 = câmara cilíndrica de Teflon (99,4 mm de altura $\times 170 \mathrm{~mm}$ de diâmetro externo); 4 = cartucho contendo adsorvente sólido (Porapack) para coleta dos COVs emitidos pela planta; $5=$ bomba de baixa vazão para sucção do ar).
Análise cromatográfica de COVs oxigenados - Os padrões de álcoois utilizados na cromatografia a gás foram obtidos da Research Chemical. Soluções estoques de padrões individuais (1-hexanol, 1-pentanol, 3-metil-1-butanol, 2-pentanol, 2-metil-2-butanol, 4-penten-1-ol, 2-pente-1-ol, 3-hexen-1-ol, 1-octen-3-ol, 4-penten-2-ol, 1-penten-3-ol, 2-metil-3-buten-2-ol, 3-metil-2-buten-1-ol, hexanal, linalool e mentol) foram preparadas em metanol, grau cromatográfico, da Merck na concentração de 1.000 mg.L ${ }^{-1}$ Misturas de 50 e $100 \mathrm{mg} . \mathrm{L}^{-1}$ foram obtidas a partir das soluções estoques individuais.

Soluções diluídas de hidrazonas dos compostos carbonílicos preparadas em acetonitrila (grau cromatográfico, Merck), a partir da mistura padrão (formaldeído, acetaldeído, acetona, acroleína, propanaldeído, crotonaldeído, 2-butanona, metacroleína, benzaldeído, valeraldeído, tolualdeído e hexaldeído, adquirida pela Radian International) foram utilizadas na cromatografia a líquido. Uma mistura de solventes contendo acetonitrila, tetrahidrofurano (grau cromatográfico, Merck) e água deionizada foi utilizada como eluente.

As soluções utilizadas na cromatografia de íons foram preparadas com água deionizada (Nanopure Water System, Barnsted). Soluções-estoque dos padrões individuais de ácidos carboxílicos (ácidos fórmico, acético, glicólico, láctico, glioxílico e pirúvico) foram preparadas na concentração de $100 \mathrm{mg}$. $\mathrm{L}^{-1}$. Uma solução $25 \mathrm{mmol} . \mathrm{L}^{-1} \mathrm{de}$ $\mathrm{Na}_{2} \mathrm{~B}_{4} \mathrm{O}_{7}$ foi utilizada como eluente.

A análise de álcoois foi feita utilizando um cromatógrafo a gás acoplado a um espectrômetro de massas (modelo QP5050A, Shimadzu). Para a separação foi empregada a coluna analítica DB5-MS (50 $\mathrm{m} \times 0,2$ $\mathrm{mm} \times 0,33 \mu \mathrm{m})$. A temperatura do injetor foi de $260{ }^{\circ} \mathrm{C}$ e a interface entre o cromatógrafo e a fonte de íons foi mantida à temperatura de $240{ }^{\circ} \mathrm{C}$. A tensão do detector foi de $2,75 \mathrm{KV}$ e os íons produzidos foram monitorados no modo de detecção SCAN.

Os compostos carbonílicos foram analisados em um cromatógrafo a líquido de alto desempenho (modelo LC-10AD, Shimadzu), acoplado a um detector espectrofotométrico $(\lambda=360 \mathrm{~nm})$. A separação foi feita com uma coluna da Supelco LC18 $(25 \mathrm{~cm} \times 4,6 \mathrm{~mm} \times 5 \mu \mathrm{m})$ empregando programa de gradiente. Um volume de $20 \mu \mathrm{l}$ foi injetado na coluna analítica através de uma válvula de injeção Rheodyne.

Os ácidos carboxílicos foram analisados em um cromatógrafo de íons (modelo 4000i, Dionex) acoplado a um detector de condutividade elétrica (sensibilidade de 3 a $30 \mu \mathrm{S}$ ) e um sistema de supressão química. Uma pré-coluna ATC-1 foi instalada antes da válvula de injeção para eliminação de eventuais interferentes no eluente. A separação dos ácidos foi efetuada por uma coluna AS11 e a diminuição da condutividade do eluente foi feita empregando uma coluna supressora AMMS-II e uma solução $12,5 \mathrm{mM}$ de $\mathrm{H}_{2} \mathrm{SO}_{4}$ como regenerante. 


\section{Resultados e Discussão}

Taxas de emissão específicas para cada espécie de planta são dados importantes para estabelecer o inventário de emissões biogênicas. Tais taxas têm sido determinadas somente para um número muito limitado de espécies de plantas (Hewitt \& Street 1992).

A caracterização química das emissões biogênicas, uma mistura altamente complexa, é um desafio analítico e a instrumentação necessária para identificar e quantificar os compostos biogênicos nem sempre está disponível. Por exemplo, a quantificação dos compostos emitidos exige sistemas de pré-concentração para o enriquecimento da amostra antes da análise cromatográfica, como a criogenia ou a retenção em adsorventes sólidos com dessorção térmica dos componentes.

No presente estudo, os compostos voláteis emitidos por $F$. benjamina foram caracterizados qualitativamente por diferentes técnicas cromatográficas, entretanto, a quantificação dos mesmos, não foi possível.

Apesar de o sistema estático de coleta do ar não ser freqüentemente utilizado na avaliação das emissões de plantas devido às possíveis interferências provocadas pelo estresse da planta (Monson et al. 1995), esse sistema foi inicialmente testado por estar disponível em nosso laboratório. Os COVs oxigenados identificados nos experimentos em câmara estática foram o ácido fórmico, ácido acético, formaldeído, acetaldeído, hexanal e mentol. Na figura 3 estão apresentados os cromatogramas e os respectivos picos identificados por cromatografia de íons (A), cromatografia a líquido (B) e cromatografia a gás $(C)$. A detecção dessas espécies foi possível apenas quando a planta permaneceu durante 90 min na câmara estática. Um tempo de permanência longo implica em submeter à planta a um estresse, relacionado às mudanças na natureza qualitativa $\mathrm{e}$ quantitativa das emissões e, eventualmente, podem ocorrer emissões de compostos, que muitas vezes, não seriam emitidos se a mesma estivesse em seu hábitat natural (Monson et al. 1995). Os estudos realizados empregando esse sistema, entretanto, podem ser úteis para uma avaliação preliminar dos compostos presentes nas emissões de plantas (Sharkey 1996).

Segundo Kesselmeier \& Staudt (1999), os experimentos para avaliação das emissões de COVs por plantas em câmara dinâmica ("cuvette") causam menor estresse à planta, uma vez que não há interrupção do fluxo de água, minimizando eventuais artefatos de coleta. Nesse sistema, um fluxo de ar limpo penetra na câmara, arrasta os gases orgânicos emitidos pela planta e sai da câmara passando diretamente pelos adsorvedores. De modo diferente do sistema estático, a pré-concentração dos gases emitidos no sistema dinâmico ocorre imediatamente à saída do ar na câmara (pré-concentração "on-line"). Também, no sistema dinâmico, é menor a adsorção dos gases orgânicos na superfície interna da câmara.
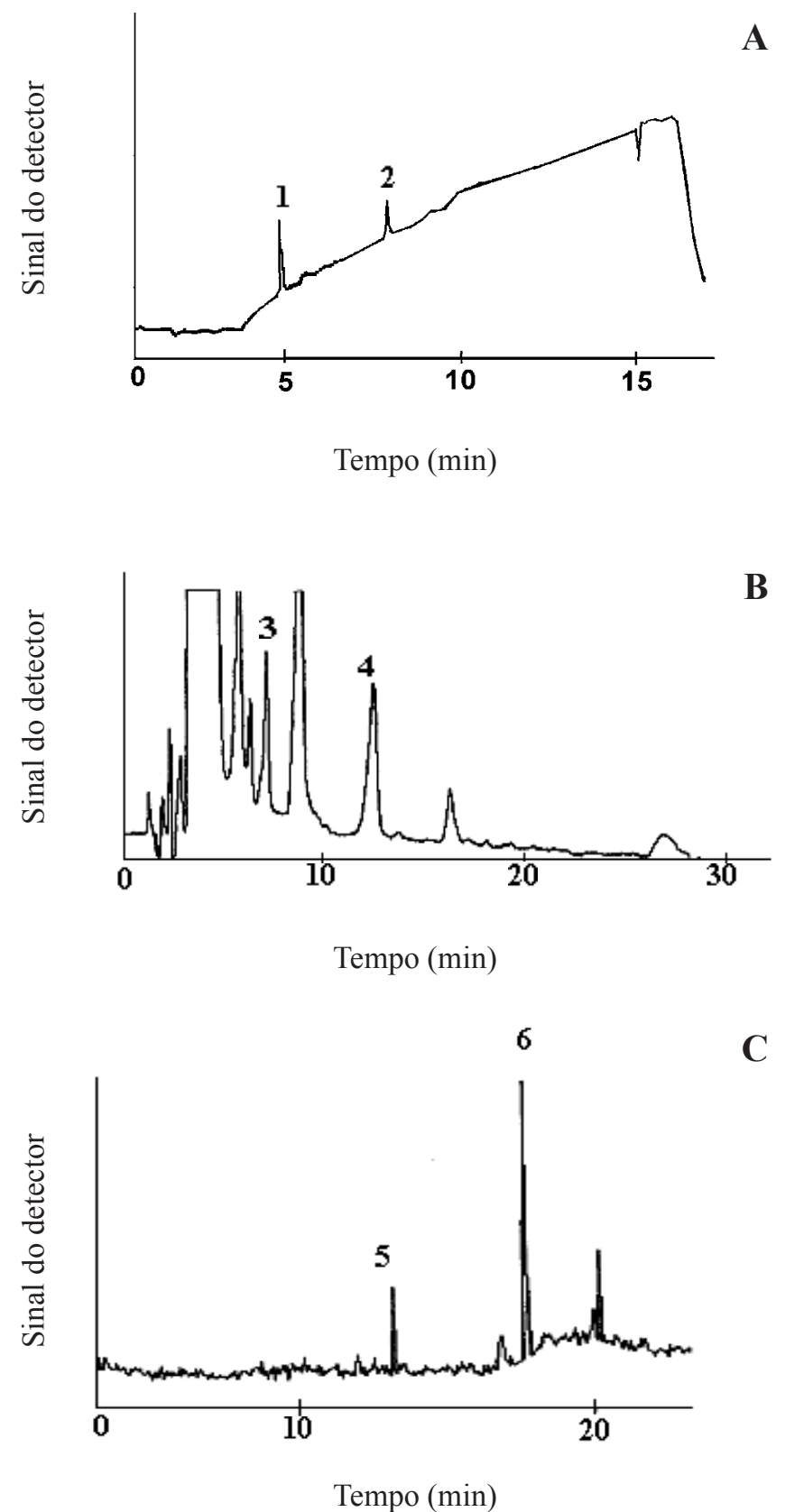

Figura 3. Cromatogramas dos gases emitidos por Ficus benjamina empregando-se o sistema estático, obtidos por cromatografia de íons (A), cromatografia a líquido (B) e cromatografia a gás $(\mathrm{C})(1$ ácido acético; 2 = ácido fórmico; $3=$ formaldeído; $4=$ acetaldeído; $5=$ hexanal; $6=$ mentol). Os picos não numerados representam espécies não identificadas. 
Os experimentos em câmara fechada dinâmica permitiram identificar álcoois (1-butanol,1-pentanol, 4-penten-2-ol, 1-penten-1-ol, linalool e mentol) e aldeído (hexanal). O cromatograma do extrato em diclorometano, obtido por cromatografia a gás, está apresentando na figura 4. Uma comparação entre os resultados obtidos pelos sistemas estático e dinâmico não é possível, uma vez que os procedimentos analíticos empregados são distintos.

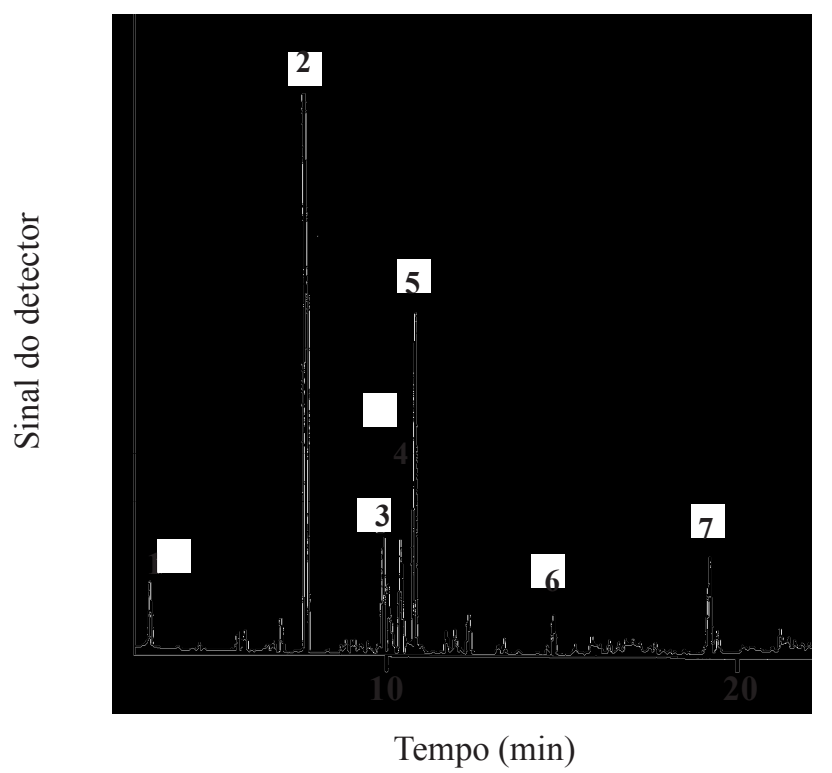

Figura 4. Cromatograma dos gases emitidos por Ficus benjamina, obtido empregando-se o sistema dinâmico, por cromatografia a gás. ( $1=4$-penten-2-ol; $2=1$ - butanol; $3=$ 1-pentanol; $4=2$-penten-1-ol; $5=$ hexanal; $6=$ linalool; $7=$ mentol).

As emissões dos compostos identificados nesse trabalho foram relatadas também para outras espécies de plantas encontradas em diferentes regiões do mundo (Konig et al. 1995, Riemer et al. 1998). As famílias Pinaceae, Fagaceae e Betulaceae, por exemplo, têm se destacado na emissão de ácidos orgânicos, sendo ácido fórmico e ácido acético os mais abundantes. A maioria das plantas emite compostos carbonílicos, tais como formaldeído, acetaldeído e hexanal (Kesselmeier \& Staudt 1999). Várias espécies de plantas emitem quantidades significativas de álcoois (Monson et al. 1995), e suas taxas, algumas vezes, superam as taxas de emissões de isopreno e terpenos. Uma grande variedade de álcoois, tais como 1-hexanol, 1-pentanol, 3-metil-1-butanol, 2-pentanol, 2-metil-2-butanol, 4-penten-1-ol, 2-penten-1-ol, 3-hexen-1-ol, 1-octen-3-ol, 4-penten-2-ol, 1-penten-3-ol, 2-metil-3-buten-2-ol, 3-metil-2-buten-1-ol, linalool e mentol foram identificados em diferentes plantas encontradas na Califórnia (Konig et al. 1995).

Dentre os compostos identificados nas emissões de Ficus benjamina, alguns são reconhecidamente importantes na química da atmosfera por serem considerados precursores de ozônio e de outros poluentes tóxicos presentes na atmosfera. Ácidos carboxílicos e aldeídos participam ativamente das reações químicas que ocorrem no "smog" fotoquímico, episódio freqüente em atmosferas poluídas de grandes centros urbanos, como São Paulo. Além disso, os ácidos orgânicos são responsáveis pelo aumento da acidez atmosférica em áreas urbanas e florestais.

Apesar de os resultados qualitativos obtidos no presente trabalho serem importantes, investigações futuras sobre emissões de plantas para incluir os numerosos COVs oxigenados se fazem necessárias. No momento, existe pouca disponibilidade de dados de emissão para muitos dos COVs oxigenados e as estimativas de emissão desses compostos são bastante imprecisas. Como as espécies de plantas e a qualidade/ quantidade de emissão são assuntos ainda em debate, dirigir pesquisas para esse campo é fundamental.

Agradecimentos - Os autores agradecem à Fapesp pelo apoio financeiro à pesquisa (processo n. 99/06683-3) e pela bolsa de pós-doutorado concedida à Silvia Ribeiro de Souza (processo n. 98/11079-9), e à Silvana Odete Pisani pela valiosa colaboração na revisão do manuscrito.

\section{Referências bibliográficas}

ARAGAKI, S. \& MANTOVANI, W. 1998. Caracterização do clima e da vegetação de remanescente florestal no Planalto Paulistano (SP). In Anais do IV Simpósio sobre Ecossistemas Brasileiros (S. Watanabe, coord.). Aciesp, São Paulo, v. 2, p.25-36.

AREY, J., WINER, A., ATKINSON, R., ASCHMANN, S.M., LONG, W.D. \& MORRISON, C.L. 1991. The emission of (Z)-3-hexen-1-ol, (Z)-3-hexenylacetate and other oxygenated hydrocarbons from agricultural plant species. Atmospheric Environment 25:1063-1075.

CICCIOLI, P. 1993. Chemistry and analysis of volatile organic compounds in the environment (H.J.T. Bloemen \& J. Burn, eds.), Blackie Academic \& Professional, London, p.92-174.

FEHSENFELD, F., CALVERT, J., FALL, R., GOLDAN, P., GUENTHER, A., HEWITT, N., LAMB, B., LUI, S., TRAINER, M., WESTBERG, H. \& ZIMMERMAM, P. 1992. Emissions of volatile organic compounds from vegetation and the implications for atmospheric chemistry. Global Biogeochemical Cycles 6:389-430. 
GROSJEAN, D. 1995. Atmospheric chemistry of biogenic hydrocarbon: relevance to the Amazon. Química Nova 18:184-201.

GUENTHER, A., HEWITT, C.N., ERICKSON, D., FALL, R., GERON, C., GRAEDEL, T., HARLEY, P., KLINGER, L., LERDAU, M., MCKAY, W.A., PIERCE, T., SCHLES, B., STEINBRECHER, R., TALLAMRAJU, R., TAYLOR, J. \& ZIMMERMAM, P. 1995. A global model of natural volatile organic compound emissions. Journal of Geophysical Research 100:8873-8892.

GUENTHER, A. 1999. Modeling biogenic volatile organic compound emissions to the atmosphere. In Reactive hydrocarbons in the atmosphere (C.N. Hewitt, ed.). Academic Press, London, p.97-118.

HARLEY, P., FRID-STROUD, V., GREENBERG, J., GUENTHER, A. \& VASCONCELLOS, P.C. 1998. Emission of 2-methyl-3-buten-2-ol by pines: a potentially large natural source of reactive carbon to the atmosphere. Journal of Geophysical Research 103:25479-25486.

HEWITT, C.N. \& STREET, R.A. 1992. A qualitative assessment of the emission of non-methane hydrocarbon compounds from the biosphere to the atmosphere in the UK: present knowledge and uncertainties. Atmospheric Environment 26:3069-3077.

KESSELMEIER, J. \& STAUDT, M. 1999. Biogenic volatile compounds (VOC): an overview on emission, physiology and ecologic. Journal of Atmospheric Chemistry 33:23-88.

KLINGER, L.F., GREENBERG, J., GUENTHER, A., TYNDALL, G., ZIMMMERMAN, P., BANGUI, M.M., MOUTSAMBOTÉ, J.M. \& KENFACK, D. 1998. Patterns in volatile organic compound emission inventories. Atmospheric Environment 29:2989-3002.

KONIG, G., BRUNDA, M., PUXBAUM, H., HEWITT, C.N. \& DUCKHAM, C.S. 1995. Relative contribution of oxygenated hydrocarbons to the total biogenic VOC emissions of selected mid-European agricultural and natural plant species. Atmospheric Environment 29:861-874.

MACDONALD, R.C. \& FALL, R. 1993a. Acetone emission from conifer buds. Phytochemistry 34:991-994.
MACDONALD, R.C. \& FALL, R. 1993b. Detection of substantial emissions of methanol from plants to the atmosphere. Atmospheric Environment 27:1709-1713.

MONSON, R.K., LERDAU, M.T., SHARKEY, T.D., SCHIMEL, D.S. \& FALL, R. 1995. Biological aspect of constructing volatile organic compound emission inventories. Atmospheric Environment 29:2989-3002.

NEMECEK-MARSHALL, M., MACDONALD, R.C. \& FALL R. 1995. Methanol emissions from leaves. Enzymatic detection of gas-phase methanol and relation of methanol fluxes to stomata conductance and leaf development. Plant Physiology 108:1359-1368.

RIEMER, D., POS, W., MILNE, P., FARMER, C., ZIKA, R., APEL, E., OLSYNA, K., KLIENDIENST, T., LONNEMAN, W., BERTMAN, S., SHEPSON, P. \& STARN, T. 1998. Observations of non-methane hydrocarbons and oxygenated volatile organic compounds at a rural site in the southeastern United States. Journal of Geophysical Research 103:2811-2812.

ROSSI, L. 1994. A flora arbustivo-arbórea da mata da Reserva da Cidade Universitária "Armando de Salles Oliveira" (São Paulo, Brasil). Boletim do Instituto de Botânica 9:1-105.

SHARKEY, T.D. 1996. Emission of low molecular mass hydrocarbons from plants. Trends Plant Science 1:78-82.

SIENFELD, J.H. \& PANDIS, S.N. 1998. Atmospheric chemistry and physics: from air pollution to climate change. John Wiley \& Sons, New York, p.234-336.

TALBOT, R. 1990. Sources and sinks of formic, acetic and pyruvic acids over Central Amazonian in wet season. Journal of Geophysical Research 95:16799-16811.

TINGEY, D.T., TURNER, D.P. \& WEBER, J.A. 1991. Factors controlling the emissions of monoterpenes organic compounds. In Trace gas emissions by plants (T.D. Sharkey, E. Holland \& H. Mooney, eds.). Academic Press, San Diego, p.93-115.

WINER, A.M., AREY, J., ATKINSON, R., ASCHMANN, S., LONG, W.D, MORRISON, C.L., \& OLSZYK, D. 1992. Emission rates of organic from vegetation in California's Central Valley. Atmospheric Environment 26:2647-2659. 